Histoire Épistémologie Langage

\section{Histoire Épistémologie Langage}

43-2 | 2021

Linguistique psychologique et sémiotique : le contexte allemand et son influence

\title{
Le tașrif des grammairiens arabes. Une morphophonologie sans morphèmes
}

\section{Georges Bohas}

\section{OpenEdition}

\section{Journals}

Édition électronique

URL : https://journals.openedition.org/hel/1319

DOI : 10.4000/hel.1319

ISSN : 1638-1580

Éditeur

Société d'histoire et d'épistémologie des sciences du langage

Édition imprimée

Date de publication : 31 décembre 2021

Pagination : 169-182

ISBN : 9791091587150

ISSN : 0750-8069

Référence électronique

Georges Bohas, "Le tașiif des grammairiens arabes. Une morphophonologie sans morphèmes 》, Histoire Épistémologie Langage [En ligne], 43-2 | 2021, mis en ligne le 01 décembre 2021, consulté le 17 décembre 2021. URL : http://journals.openedition.org/hel/1319; DOI : https://doi.org/10.4000/hel. 1319

HEL is licensed under a Creative Commons Attribution-NonCommercial-NoDerivatives 4.0 International License 


\section{LE TASTRIF DES GRAMMAIRIENS ARABES \\ UNE MORPHOPHONOLOGIE SANS MORPHÈMES}

\section{Georges Bohas}

ENS de Lyon, Interactions, corpus, apprentissage, représentations (UMR 5191, Icar), Lyon, France

\begin{abstract}
Résumé - La phonologie des grammairiens arabes (GA) et le cadre standard de la phonologie générative qui, fondée sur Chomsky \& Halle (1968), appartient désormais elle aussi à l'histoire, ont en commun de poser des représentations abstraites ('aṣl pour les GA, représentations phonologiques pour les générativistes) et des règles afin de rendre compte des alternances présentées par les mots. Du fait que les GA n'ont pas élaboré le concept de morphème et se sont limités au mot surgissent des différences considérables dans l'organisation de la phonologie. S'ajoute à cela le fait que les règles des GA ne sont pas markoviennes et ne s'appliquent pas mécaniquement, mais en vertu de grands principes portant sur la langue ou les intuitions des locuteurs (les Arabes anciens mythiques pour les GA).
\end{abstract}

Mots-clés - grammairiens arabes, morphologie, phonologie, règles
Abstract - Both the phonology of the Arab grammarians (AG) and the standard framework of generative phonology, which - based as it is on Chomsky \& Halle (1968) - is now also a part of history, have in common the positing of abstract representations ('aṣl for the former, and phonological representations for the latter) and rules to account for alternations present in words. The fact that the AG did not elaborate the concept of the morpheme has resulted in considerable differences in the organization of phonology. Added to this is the fact that the rules of AG are not Markovian and do not apply mechanically, but by virtue of grand principles about the language or the intuitions of the speakers (the mythical Ancient Arabs for $A G)$.

Keywords - Arab grammarians, morphology, phonology, rules

\section{INTRODUCTION}

Pour les grammaires orientalistes traditionnelles, le verbe arabe est le composé d'un radical et de préfixes ou suffixes. Wright (1971 [1859-1862] : 53-55) parle de suffixed et prefixed pronouns. Pour l'accompli, Blachère \& GaudefroyDemombynes expliquent : « il y a donc un radical auquel s'ajoutent des suffixes » (1975 [1937] : 39) et «l'inaccompli est formé à l'aide de préfixes et de suffixes » (ibid. : 41). Dans un des derniers avatars de ces grammaires à but pédagogique on trouve de même : "La conjugaison de l'accompli est une conjugaison dite "à suffixes" : on suffixe des marqueurs de personnes, de nombre et de genre au radical verbal » (Sartori 2017 : 24). Quant à l'inaccompli, il est « premièrement une conjugaison à préfixes et secondairement à suffixes » (ibid. : 26). 
En grammaire générative, dans la thèse de Brame (1970:4) rédigée dans le cadre standard (Chomsky \& Halle 1968), il en va de même : le verbe à l'accompli est formé d'un radical (stem) symbolisé par X et de suffixes (person markers), ce qui donne le tableau suivant :

\begin{tabular}{|c|c|c|c|c|c|c|c|c|c|c|c|}
\hline \multicolumn{4}{|c|}{ Singular } & \multicolumn{4}{|c|}{ Plural } & \multicolumn{4}{|c|}{ Dual } \\
\hline$X$ & tu & I & & $\mathrm{X}$ & nā & we & & & & & \\
\hline$X$ & ta & you & $\mathrm{m}$. & $X$ & tum & you & $\mathrm{m}$. & $X$ & tumā & you & $\mathrm{m}$. \\
\hline $\mathrm{X}$ & $\mathrm{ti}$ & you & f. & $\mathrm{X}$ & tunna & you & f. & $X$ & tumā & you & f. \\
\hline$X$ & $\mathrm{a}$ & he & & $X$ & $\overline{\mathrm{u}}$ & they & $\mathrm{m}$. & $X$ & $\overline{\mathrm{a}}$ & they & $\mathrm{m}$. \\
\hline $\mathrm{X}$ & at & he & & $X$ & na & they & f. & $X$ & atā & they & f. \\
\hline
\end{tabular}

Cette division en morphèmes : radicaux et suffixes n'a rien à voir avec celle que l'on trouve dans la morphophonologie (tașrīf) des grammairiens arabes, laquelle ignore complètement les concepts de «radical» et de «suffixe», autrement dit, dans la morphophonologie des grammairiens arabes (désormais GA), la notion de morphème n'a pas cours. Comme nous l'avons démontré dans Bohas (1979), pour les GA, le découpage proposé par ce tableau (et effectué aussi par les grammaires orientalistes traditionnelles) n'a aucun sens car, pour eux, la notion de radical n'existant pas, un verbe comme kataba ${ }^{1}$ n'est pas analysable. Kataba est la forme verbale minimale et c'est à elle que sont adjoints $t u, t a, t i$ qui ne sont pas des marqueurs grammaticaux, mais des noms, plus précisément des pronoms sujets, le $w$ et le $n \bar{a}$ du pluriel également. Le verbe et son pronom sujet constituent comme un seul mot. Leur analyse est donc ${ }^{2}$ :

\begin{tabular}{|l|l|}
\hline Verbe & Sujet \\
\hline katab $a$ & tu \\
\hline kataba & ta \\
\hline kataba & ti \\
\hline kataba & na" \\
\hline
\end{tabular}

On obtient dans tous ces cas une succession de quatre lettres mues (CV) (mutaharrik $=$ suivi de voyelle ${ }^{3}$ ), et une des grandes règles qui, selon les GA,

1 Seules les formes attestées phonétiquement, « représentations phonétiques » dans la terminologie générativiste, sont écrites en italique. Nous parlerons souvent à leur sujet de formes concrètes. Les autres sont en romain.

2 Rappelons que pour les GA les voyelles que nous appelons longues $(\bar{a}, \overline{1}, \bar{u})$ sont conçues comme des brèves avec un segment allongeant $\overline{\mathrm{u}}=\mathrm{uw}, \overline{\mathrm{i}}=$ iy et $\overline{\mathrm{a}}=\mathrm{a}$ ". J'utilise ce symbole " pour noter le alif des GA, allongeant du a. Brame argumente en faveur d'une représentation/uw/ et/iy/ des voyelles longues pour $\bar{u}$ et $\overline{1}(1970: 100-105)$.

3 Dans le cas contraire, la lettre qui n'est pas suivie de voyelle est dite «sākin » : quiescente. Pour les GA d'autrefois et pour les maîtres d'école d'aujourd'hui dans le monde arabe, les éléments phonétiques minimaux sont : lettre mue et lettre quiescente. Le concept de syllabe n'existe pas et au cours de ma carrière d'enseignant de la métrique arabe, $\mathrm{j}$ 'ai pu constater combien il était difficile pour les étudiants formés à l'analyse mue/quiescente d'acquérir la notion de syllabe, sauf pour ceux qui sont passés par des écoles 
commandent la structure des mots est qu'une séquence de quatre $\mathrm{CV}$ est interdite (Bohas 1979 : 43). Écrivons cette interdiction comme suit : *4CV ${ }^{4}$. C'est à cette interdiction qu'est attribuée l'élimination des $a$ en italique dans le tableau pour donner katabtu, katabta, etc.

Pour le pluriel masculin l'analyse sera aussi :

\begin{tabular}{|l|l|}
\hline Verbe & Sujet \\
\hline kataba $a$ & w \\
\hline
\end{tabular}

Dans ce cas, le $a$ se transforme en $u$ pour s'harmoniser (munāsaba) avec le $w$ et donner katabuw (pour nous katabū). Toutes ces opérations sont résumées dans al-Ushmūni (s. d. : I, 58) :

Quant à darabtu, intalaqna", istaqbalna", l'effacement de la dernière voyelle [de la base verbale : daraba tu, intalaqa na", istaqbala na"] est accidentel, dû à l'application obligatoire d'une règle motivée par la répugnance pour la suite $* 4 \mathrm{CV}$ dans ce qui est comme un seul mot, car le pronom sujet est comme une partie de son verbe. De même le $u$ de darabuw est accidentel et ce qui l'a rendu obligatoire, c'est la nécessité d'harmoniser la voyelle [i.e., le a du verbe ḍaraba w] avec le $w$ qui suit ${ }^{5}$.

Pour notre auteur, une voyelle appartenant à la structure de base est dite « lāzim » « intrinsèque ou inhérente » et la voyelle introduite par règle est conçue comme «'árịd» «accidentelle». Dans daraba $\mathrm{w}$, le a est intrinsèque et le $u$, introduit par harmonie avec le $w$, est « accidentel».

Enfin, la forme katabat ( $3^{\mathrm{e}}$ pers. fém. sg.) s'analyse en :

\begin{tabular}{|l|l|}
\hline Verbe & $\mathrm{t}$ du féminin \\
\hline kataba $a$ & $\mathrm{t}$ \\
\hline
\end{tabular}

Ce qui donne directement katabat, puisque l'interdiction $* 4 \mathrm{CV}$ ne peut pas opérer ici.

Cela suffit à montrer qu'une organisation avec morphème (orientalistes et Brame) est bien différente d'une organisation sans morphème (GA) qui ne connaît que le verbe (comme kataba), le nom (plus précisément ici le pronom, comme $t u$ ), la lettre (comme $y$, lettre de la ressemblance) et la voyelle de l'i 'rāb (flexion, comme $u$ de yaktubu à l'inaccompli que nous étudierons plus loin).

Certes, tous, les orientalistes et Brame, ont recours à la notion de racine comme allant de soi, pour l'arabe comme pour les autres langues sémitiques : « La langue arabe est une langue sémitique dite à racines» (Sartori 2017 : 11); mais quand deux théories font usage de termes techniques analogues cela ne doit pas amener

occidentales, anglaises ou françaises, où ce concept a cours. Ce qui montre que le concept de syllabe n'est pas inné mais acquis à l'école.

4 En métrique arabe, les séquences de brèves $\cup(=\mathrm{CV})$ sont restreintes par la contrainte que les métriciens arabes appellent mu 'āqaba et que nous avons formulée dans Bohas \& Paoli (1997 : 57) comme suit : toute séquence de brèves égale ou supérieure à 2 (quelle que soit son origine) figurant à gauche d'un watid doit appartenir au même pied que lui.

5 Toutes les traductions de l'arabe sont les nôtres. 
à croire que l'organisation des deux théories soit identique; autrement dit, qu'elles font un même usage de ces termes techniques. Ainsi, les grammairiens arabes font usage des termes aṣl= racine et binya ou wazn $=$ schème, termes techniques repris par les grammaires orientalistes de l'arabe (Wright 1971 [18591862]), de l'hébreu (Touzard 1949 [1905]) et du syriaque (Duval 1881) (entre autres) qui toutes utilisent les mêmes abréviations fondées sur les multiples formes du verbe $f a^{\prime} a l^{6}{ }^{6}$ pour désigner le schème et distinguer ce qui fait partie de la racine et ce qui lui est adjoint ; repris également par les linguistes structuralistes dans la ligne de Cantineau (1950a et b) et les générativistes dans la ligne de McCarthy (1979), mais il ne faudrait pas en conclure pour autant que l'organisation de la morphologie soit la même dans les quatre cas.

As shown in Bohas (1979) published in Bohas \& Guillaume (1984:162-166), the AGs views on word-formation are quite different from root-and-pattern morphology whereby every noun or verb is derived independently of each other as the result of the mapping of a consonantal root onto a pattern as in e. g. Cantineau (1950a, b) or McCarthy (1979). (Bohas \& Lowenstamm, à paraître)

Pour les grammairiens arabes en effet, les mots sont dérivés d'autres mots. Les seuls qui soient formés directement sur la racine sont les bases nominales et les mașdars (selon l'école bașrienne majoritaire). Le verbe accompli (māḍ̂l) est formé sur le mașdar, l'inaccompli (mudāri " le ressemblant») sur l'accompli, le participe actif sur l'inaccompli, etc. Cette organisation, largement développée dans Bohas (1979, première partie), résumée dans un tableau (Bohas 1979 : 240 ; Bohas \& Guillaume 1984 : 163) a été reformulée dans Bohas \& Lowenstamm (à paraître). Ainsi conçue, la morphologie constitue la première partie du tașrîf des GA, celle qui «porte sur les lois générales permettant de connaître les [divers] états des structures des mots » (Ibn al-Hāăib 1975 [1939] : I, 1).

La deuxième partie du tașrîf, qui décrit les changements de ces structures pour des raisons phonétiques (Ibn 'Ușfür 1970 : 32), n'est pas sans analogie avec la phonologie générative, cadre standard (Chomsky \& Halle 1968), appliquée à l'arabe par Brame (1970). Dans les deux cas, on pose des représentations abstraites « phonologiques », «'așl » pour les GA, dont dérivent les représentations concrètes «phonétiques » via l'application de règles. Ajoutons que pour les GA, ces représentations abstraites sont analogiques (qiyāsiyya), c'est-à-dire que toute représentation abstraite incluant un $w$ ou un $y$ (consonnes « malades » auxquelles des règles vont s'appliquer) doit être analogue à une forme effectivement attestée incluant à la place du $w$ ou $y$ en question une consonne « saine ». Comme nous l'avons remarqué dans Bohas et Lowenstamm (à paraître) :

There is a major difference, however. A generative phonologist trying to identify an underlying representation faces an open array of options ranging from the most concrete to the most abstract ${ }^{7}$. The final determination will be made on the basis of metagrammatical considerations such as consistency, economy, elegance, a principled position wrt to abstractness, etc. The AGs, by contrast, are not facing

6 Où $\mathrm{f}=$ première consonne, ${ }^{\prime}=$ deuxième consonne et $\mathrm{l}=$ troisième consonne.

7 For an example of extreme abstractness, see Lightner (1979) and Kiparsky (1973) for a plea against excessive abstractness. 
a similarly unlimited range of options. Indeed, the identity of underlying phonological representations is specified in precise fashion by the system itself, viz. their makeup as they exit tașrif $I$. Thus, any verbal form (no matter how opaque on the surface) goes back by construction to that level: to fa'ala, facila, or fa'cula if Perfective; to yaf'ulu, yaf'alu or yaf'ilu if Imperfective; to fa"il in the case of an agentive participle, etc.

Dans cette perspective, la grammaire des GA et la théorie proposée par Brame sont comparables, néanmoins, il est une différence qui n'a pas été assez approfondie jusqu'ici ${ }^{8}$, celle qu'implique sur l'application des règles l'absence du concept de morphème (radical, préfixe, suffixe) dans la conjugaison des verbes dits " défectueux » comme da' $\bar{a}$ (appeler), ramā (jeter) ( $d a^{\prime} a^{\prime}$ " et rama" dans la notation des GA) et radiya (être satisfait). Nous allons donc revenir sur cette question en nous fondant particulièrement sur le tome II du Mumti 'd'Ibn 'Usfür, grammairien andalou du XIII ${ }^{\mathrm{e}}$ siècle (1200-1271).

\section{L’ACCOMPLI}

Comme nous l'avons dit, pour Brame le verbe à l'accompli est formé d'un radical (stem) qu'il symbolise par X et de suffixes (person markers) (voir le tableau cidessus) ; dans ces conditions kataba sera analysé en katab+a, katabat en katab+at, et katabtu en katab+tu; autrement dit radical + suffixe. Les représentations phonologiques de ramā et ramat seront analysées de même:/ramay+a/ et /ramay+at/. S'appliqueront trois règles ordonnées :

GL Glide Elision

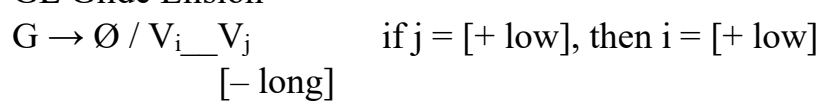

TR Truncation

$$
\mathrm{v} \rightarrow \varnothing / \mathrm{V}_{-} \mathrm{C}\left\{\begin{array}{l}
\# \\
\mathrm{C}
\end{array}\right\}
$$

Lengthening

$$
\begin{aligned}
\mathrm{V}_{\mathrm{i}} \mathrm{V}_{\mathrm{i}} \rightarrow & \mathrm{V}_{\mathrm{i}} \\
& {[+ \text { long }] }
\end{aligned}
$$

Ce qui donne :

\begin{tabular}{|l|l|l|}
\hline & $/$ ramay+a\#/ & $/$ ramay+at\#/ \\
\hline GL & $/$ ramaØ+a\#/ & $/$ ramaØ+at\#/ \\
\hline TR & & $/$ ramaØ+Øt\#/ \\
\hline Length & /ramā\#/ & \\
\hline & {$[$ ramā] } & [ramat] \\
\hline
\end{tabular}

C'est là qu'apparaît une différence fondamentale entre les deux organisations.

8 À notre connaissance, du moins 
Pour une forme comme katabatā (duel $3^{\mathrm{e}}$ pers. fém.), Brame, analysant en morphèmes, va partir d'une représentation $/ \mathrm{katab}+\mathrm{at}+\overline{\mathrm{a}} /$ et pour ramatā il va partir de ramay $+\mathrm{at}+\overline{\mathrm{a}}$, et appliquer des règles pour dériver la représentation phonétique. Pour les GA, au contraire, des règles vont s'appliquer d'abord à ramaya pour donner rama", ensuite, on ajoute le $t$ du féminin pour donner une nouvelle représentation abstraite : rama"t, forme à laquelle s'applique une autre loi générale que nous formulons ainsi : ${ }^{2} 2 \mathrm{Q}$ : la langue arabe ne tolère pas la succession de deux quiescentes. "t constituent une succession de deux quiescentes, le alif et le $t$. On éliminera donc le alif pour obtenir ramat. Ensuite, on ajoutera le a" du duel pour obtenir : ramata". Il en va de même pour da 'atā. Mais observons que cette forme $\mathrm{du}$ féminin $3^{\mathrm{e}}$ pers. duel nécessite pour Brame l'introduction d'une frontière de mot dans sa représentation initiale. Il dit en effet (Brame 1970 : 447) :

The correct derivation immediately follows from the underlying sequence /da'aw+at\#aa/.

(21) /da'aw+at\#aa/

$$
\begin{array}{ll}
\text { da'a } Ø+a t \# a a & \text { Glide Elision } \\
\text { da'a+Øt\#aa } & \text { Truncation } \\
/ \mathrm{da}^{\prime}+\mathrm{at} \# \overline{\mathrm{a}} / & \text { Lengthening }
\end{array}
$$

Sans la présence de cette frontière de mot, la règle de troncation ne pourrait pas s'appliquer et sa grammaire dériverait la forme agrammaticale *da ātā.

Pour les GA, la représentation initiale de rama" est ramaya, analogique de daraba. À cette représentation s'applique la règle que j'ai appelée MUTA1 (Bohas 1979 : 366 sv. ; Bohas \& Guillaume $1984: 241$ sv.) formulée ainsi par Ibn Ğinnī (dans Ibn Ya īšs 1973, Šarh al-Mulūkī : 218-219 et dans Ibn Ğinnī 1960, alMunșif: II, 116-117) :

Lorsque le w et le y se trouvent précédés d'un a et suivis d'une voyelle, ils se muent en alif (") sauf dans quelques cas exceptionnels qui laissent apparaitre la représentation sous-jacente et en constituent un indice, ou lorsqu'on craint d'aboutir à l'ambiguité ou lorsque le maintien du glide constitue un signal.

Dans le formalisme standard de la phonologie générative, cela donnerait :

$$
\begin{aligned}
& \text { MUTA1 }\left\{\begin{array}{c}
\mathrm{w} \\
\mathrm{y}
\end{array}\right\} \rightarrow \text { " / a_v } \\
& \text { ramaya }>\text { MUTA1 > rama" }
\end{aligned}
$$

Pour éviter l'apparition d'une forme agrammaticale comme *rama"a, il faut se débarrasser de la voyelle du contexte de droite. On pourrait dire que du fait que, dans la logique des GA, le alif est toujours quiescent, la voyelle de droite du contexte / a_v tombe ipso facto. Ibn 'Ușfūr n'approfondit pas la question, mais, un siècle avant lui, Ibn Ya'ǐš (1158-1245) dans le Šarh al-Mulūkī l'avait fait clairement ${ }^{9}$ :

Sache que la mutation ne s'applique au w et au y qu'après qu'ils aient été affaiblis tous deux par l'effacement de la voyelle qui les suit. (Ibn Ya'îšs 1973 : 225) 
Le but de cette restriction est de permettre que la règle s'applique à qawala et ramaya mais pas à sawț (fouet) ou à šayh̆ (vieillard) dans lesquelles le $w$ et le $y$ n'ont jamais été suivis de voyelle.

On peut donc préciser la règle MUTA1 :

$$
\operatorname{MUTA1}\left\{\begin{array}{l}
\mathrm{w} \\
\mathrm{y}
\end{array}\right\} \rightarrow " / \mathrm{a} \_(\mathrm{v} \rightarrow \varnothing)
$$

Ainsi donc on parvient de ramaya à rama" $(=\operatorname{ram} \bar{a})^{10}$ et de da' awa à $d a^{\prime} a^{\prime \prime}$ $\left(=d a^{\prime} \bar{a}\right)$.

Dans la logique des GA, une fois le processus décrit, il importe d'en trouver la cause ; quelle est donc la cause de l'application de cette mutation? Ibn 'Ușfūr l'explicite ci-dessous ${ }^{11}$ :

La cause en est la réunion de la lourdeur des deux semblables, je veux dire le $a$ du 'et celui du L avec la lourdeur du $y$ ou du $w$, si bien que le y ou le w se changent en alif du fait de la légèreté du alif, et comme celui-ci n'est jamais suivi de voyelle, cela met un terme à la réunion des semblables. (Ibn 'Ușfür 1970 : 533)

Dans al-Munșif (II : 116), Ibn Ğinnī précise que $w$ et $y$ ressemblent aux voyelles (harakāt). Dans le Šarh al-Mufașșal, Ibn Ya '̄̌š explicite encore ${ }^{12}$ :

C'est que le $w$ est compté pour deux $u$ et le $y$ pour deux $i$; comme ils sont euxmêmes précédés de $a$ et suivis de voyelle, cela constitue une suite de quatre éléments semblables, et la réunion des semblables répugne au locuteur. (Ibn Ya 'īs $1886: 1363-1364)$

L'application de MUTA1 est donc motivée par la «lourdeur $»^{13}$ issue de la répétition des semblables. Le passage de ramaya à ramā est donc décrit et motivé.

À cette forme rama" (= ramā) s'adjoint le $\mathrm{t}$ du féminin pour donner : rama"t, la séquence "t constituant une suite de deux consonnes quiescentes (*2Q), le " est éliminé et l'on obtient : ramat. Cette différence d'analyse et donc de constitution du niveau initial de la dérivation et l'application cyclique des règles qui en découle constituent une différence radicale entre les deux modèles, et plus encore l'enchevêtrement des formes concrètes et des représentations abstraites, comme cela apparaît dans la dérivation de ramat qui passe par les étapes suivantes :

\begin{tabular}{|l|l|l|}
\hline abstrait & ramaya & MUTA1 \\
\hline concret & rama" & ajout de t \\
\hline concret/abstrait & rama"t & *2Q > effacement de " \\
\hline forme finale & ramat & \\
\hline
\end{tabular}

Cela est fort bien résumé dans Ibn 'Ușfūr, (1970 : 525). Comme il est bien connu, dans la pratique des GA les consonnes radicales sont figurées par celles de

10 Quand cela paraîtra utile nous mettrons entre parenthèses précédée du signe $=$ la transcription occidentale standard.

11 Voir aussi al-Munșif (II : 116).

12 Dans l'édition al-Tibā 'a al-munīriyya, tome 10, p. 19.

13 Sur cette notion voir Bohas \& Lowenstamm (à paraître). 
la racine $\mathrm{F}^{\prime} \mathrm{L}^{14}$. $\mathrm{L}$ désigne donc la troisième consonne dans le texte suivant et ailleurs.

Si à l'un de ces verbes s'adjoint le marqueur du féminin, il demeure tel quel si son L est, dans la forme phonétique, y ou w, comme dans :

saruwa saruwat i l-mar'a il/la femme est généreux/se

radiya radiyat Hind il/Hind est satisfait/e

guziya guziyat i l-a'da' il/elle a été attaqué/ $\mathrm{e}^{15}$

Mais si le L est un alif [comme dans rama"], alors ce alif est effacé à cause de la rencontre de deux quiescentes, comme dans : ramat Hind a jeté Hind.

Poursuivons avec la dérivation de ramata" (duel $3^{\mathrm{e}}$ pers. fém.) où apparaît encore mieux ce que nous avons appelé enchevêtrement abstrait/concret en passant par les étapes suivantes :

\begin{tabular}{|l|l|l|}
\hline abstrait & ramaya & MUTA1 \\
\hline concret & rama" & ajout de t \\
\hline concret/abstrait & rama" t & $* 2 \mathrm{Q}>$ effacement de " \\
\hline concret ramat & & ajout de a" (pronom sujet) \\
\hline concret/abstrait ramat a" & & \\
\hline forme finale & ramata", dans la notation occidentale : ramatā \\
\hline
\end{tabular}

Que se passe-t-il à la troisième personne du pluriel masculin : ramaw, à comparer avec katabuw/katabū ? Comme nous l'avons dit dans la note 2, Brame argumente longuement pour donner aux voyelles longues $\overline{1}$ et $\bar{u}$ une représentation analogue à celle des GA : iy et uw. La représentation phonologique de ramaw sera donc pour lui : ramay+uw et la dérivation :

\begin{tabular}{|l|l|}
\hline & /ramay+uw\#/ \\
\hline GEL & /ramaØ+uw\#/ \\
\hline TR & /rama+ + w\#/ \\
\hline Length & la règle ne peut s'appliquer \\
\hline & {$[$ ramaw $]$} \\
\hline
\end{tabular}

Rien de tel pour les GA. À partir de ramaya MUTA1 donne rama" à laquelle on ajoute le pronom w du pluriel : rama"w. "w constituant une séquence de deux quiescentes, le alif est éliminé par *2Q et on obtient ramaw. Comme le dit Ibn 'Ușfür :

Si le verbe se termine par un alif et s'il est prédiqué d'un pronom absent pluriel $\left(=3^{\mathrm{e}}\right.$ pers. pl.), on l'efface à cause de la rencontre de deux quiescentes et de l'absence d'ambigüité, comme dans gazaw et ramaw. (Ibn 'Ușfür 1970 : 527)

Explicitons. On part de ramaya et par MUTA1 on obtient rama". On ajoute alors le pronom du pluriel $3^{\mathrm{e}}$ pers. : $r a m a " w$. Pour résoudre la succession de deux

14 Bohas (1979: $251 \mathrm{sv.})$.

15 Littéralement : « elle a été attaquée les ennemis », ce pluriel étant conçu comme un féminin dans l'accord du verbe précédent. La traduction en français sera donc : « les ennemis ont été attaqués ». 
quiescentes, *2Q s'applique, on efface le alif et on obtient ramaw.

La situation se complique lorsque $t u, t a, t i, n \bar{a}$ sont adjoints au verbe car ramaytu, ramayta, ramayti, ramaynā sont tout à fait analogues à katabtu, katabti, etc. Pour Brame, rien ne se passe dans ces cas, puisque la règle GEL ne peut pas s'appliquer : /ramay+tu/ donne trivialement [ramaytu].

Pour la GA, à partir de ramaya MUTA1 donne rama" à laquelle on ajoute le pronom sujet tu : rama" tu et alors, on ramène le alif à sa base : y, ce qui donne ramaytu.

Si le verbe est en relation prédicative avec un pronom du locuteur (mutakallim) ou de l'allocutaire (muhățab) quel qu'il soit, tu ramènes le alif à sa base, y ou w, comme dans ramayta, gazawta, ramaytuma", gazawtuma", ramaytum, gazawtum, ramaytunna, gazawtunna, ramayna", gazawna". (ibid. : 528)

Pour les GA, cela ne pose pas de problème, car, pour eux, il est toujours possible de recourir à la représentation initiale. Se manifeste là une autre différence entre les deux organisations. En phonologie générative les règles sont linéairement ordonnées et markoviennes.

Au contraire, j'ai montré dans Bohas $(1979: 320)$ que pour les grammairiens une telle restriction n'existait pas : «Le fait de dire que l'élément effacé garde le statut qu'il aurait s'il était toujours présent permet, de manière évidente, à toute règle qui s'applique de "regarder en arrière", au niveau initial, ou à un niveau sous-jacent antérieur. » De plus :

Non seulement les règles peuvent "regarder en arrière", "regarder en avant" et s'appliquer en fonction de l'état de la représentation initiale ou de la représentation phonétique optimale (conspirations), mais avant d'affecter un mașdar, elles peuvent également "regarder" ce qui se passe dans le verbe, le verbe et le mașdar étant considérés comme une même classe. (Bohas 1979 : 332 ; Bohas \& Guillaume $1984: 221)$

Il en va de même dans la dérivation du duel :

Si le verbe se termine par un alif et s'il est prédiqué d'un pronom absent singulier [= troisième personne singulier $]$ il reste tel qu'il était avant comme dans Zaydun $\dot{g} a z a$ " [= $\dot{g} a z \bar{a}]$ et 'Amrun rama" [= ramā $]$.

S'il est prédiqué d'un pronom absent duel, on ramène le alif à sa base, comme dans : gazawa" et ramaya" et on n'efface pas pour cause de rencontre de deux quiescentes afin d'éviter l'ambigüité entre le duel et le singulier. (Ibn 'Ușfür 1970 : 527-528)

Explicitons. On part de ramaya et par MUTA1 on obtient rama". On ajoute alors le pronom du duel, ce qui donne rama"' ; dans cette forme apparaît la succession de deux quiescentes (les deux alif), ce qui contrevient à *2Q. Si l'on en supprimait une des deux, on obtiendrait rama" qui est la forme du singulier. Pour éviter l'ambigüité entre le singulier et le duel, le " retrouve sa forme de base $y$ : ramay" et, comme le alif ne peut qu'être précédé de $a$, on obtient ramaya" $(=$ ramaya $)$.

Enfin :

S'il est prédiqué des absentes [ $3^{\mathrm{e}}$ fém. pl.], le alif est ramené à sa base et aucune règle ne l'affecte, comme dans gazawna et ramayna. (ibid. : 528) 
Explicitons. On part de ramaya et par MUTA1 on obtient rama". On ajoute alors le pronom du pluriel $3^{\mathrm{e}}$ pers. féminin et on obtient rama"na et, comme précédemment, le alif est ramené à sa base : ramayna.

Quand le verbe se termine par y ou $\mathrm{w}$, s'il est prédiqué du pronom de l'absent [ $=3^{\mathrm{e}}$ personne $]$ ou de l'allocutaire $\left[2^{\mathrm{e}}\right.$ personne $]$ ou du locuteur $\left[1^{\mathrm{re}}\right.$ personne $]$, il reste dans son état et ne change pas, comme :

$\begin{array}{lll}3^{\text {e }} \text { masc. sg. } & \text { raḍiya } & \text { saruwa } \\ 3^{\text {e }} \text { masc. duel } & \text { raḍiya" } & \text { saruwa" } \\ 3^{\text {e }} \text { fém. pl. } & \text { rạiyna } & \text { saruwna } \\ 1^{\text {re }} \text { et } 2^{\mathrm{e}} \text { masc. sg. } & \text { raḍiytu/ta } & \text { saruwtu/ta } \\ 2^{\mathrm{e}} \text { duel } & \text { rạ̣iytuma" } & \text { saruwtuma" } \\ 2^{\mathrm{e}} \text { masc. pl. } & \text { raḍiytum } & \text { saruwtum } \\ 2^{\mathrm{e}} \text { fém. pl. } & \text { rạ̣iytunna } & \text { saruwtunna }\end{array}$

C'est que rien n'exige qu'ils changent de l'état où ils sont. (ibid.)

Explicitons. On part de radiya, on ajoute par exemple le tu pronom de la première personne (le locuteur) radiiy $a$ tu, le $a$ est éliminé par contrainte $* 4 \mathrm{CV}$, comme dans kataba tu $>$ katabtu, et l'on obtient raḍiytu (= radìtu).

Reste le cas de raduw (= raḍu). Dans une dérivation à la Brame, on posera radiiy+uw\# : radical + suffixe. On se doute bien qu'il n'en va pas de même pour les GA qui partiront de radiya, forme à laquelle sera adjoint le $\mathrm{w}$ du pluriel : radiya $\mathrm{w}$, et saruwa $\mathrm{w}$ comme pour kataba $\mathrm{w}$ (> katabuw) :

À moins que le pronom soit celui de l'absent pluriel masculin, alors, tu effaces le $\mathrm{w}$ et le $\mathrm{y}$ [avec sa voyelle] (saruwa $\mathrm{w}>$ saru $w$; radiya $\mathrm{w}>$ radi $w$ ) et tu mets un $u$ avant le $w$ du pluriel, ce qui donne saruw $(=\operatorname{saru})$ et $r a d u w(=r a d \bar{u})$, car la lettre qui le précède est forcément mue par un $u$ comme dans darabuw (daraba $\mathrm{w}>$ darabuw). (ibid. : 529)

Mais qu'est-ce qui motive cet effacement?

Si tu disais * radiiyuw et *saruwuw, tu trouverais lourd le u sur le y et le w, du fait qu'ils sont précédés par une lettre mue, et il faudrait donc l'effacer (*rạ̣iyw et *saruww) ce qui amènerait la constitution d'une suite de deux quiescentes : le w du pronom et le $\mathrm{y}$ ou le $\mathrm{w}$ qui le précèdent. Tu effaces donc ce qui précède le $\mathrm{w}$ du pronom (saru $\varnothing \mathrm{w}$, radi $\varnothing \mathrm{w}$ ) car il est plus facile d'effacer la lettre que d'effacer le pronom et tu dis saruw et pour radidya, après l'effacement, tu mets un u sur ce qui précède le $\mathrm{w}$ (radiØ $\mathrm{w}>$ radu $\mathrm{w}$ ) et tu dis raduw pour sauver le $w$ du pronom. Car si tu laissais le $\mathrm{i}($ dans raḍi $\varnothing \mathrm{w}$ ), alors le $\mathrm{w}$ du pronom se transformerait en y du fait qu'il est quiescent et précédé de $\mathrm{i}$; et tu dirais radiy ce qui amènerait à l'ambiguïté entre le singulier et le pluriel. (ibid. : 529)

Éviter l'ambiguïté est une motivation à laquelle les GA recourent souvent, nous l'avons déjà vue à l'œuvre dans la dérivation de gazawa" (= gazawā) et ramaya" (= ramayā). 


\section{L'INACCOMPLI \\ (LE RESSEMBLANT DANS LA TERMINOLOGIE DES GA)}

Nous avons vu qu'à l'accompli la dérivation partait de la forme minimale : le verbe $3^{\text {e }}$ pers. masc., il en va de même à l'inaccompli. Par exemple : yaktubu est le singulier, $y$ est la lettre de ressemblance et le $u$ final relève de la flexion casuelle. Pour former le pluriel on ajoute le pronom pluriel w(na) ce qui donne yaktubuwna. Ce « $n a »$ relève de la flexion casuelle, il est présent quand le verbe n'est ni au subjonctif ni à l'apocopé. Pour yaġzū et yarmī on partira de yagzuwu et yarmiyu, analogues à yaktubu et yadribu. Pourquoi ces formes de base sont-elles sujettes au changement? «On efface le u car il est ressenti comme lourd sur le y et le w, car avec le w ils ont le statut de deux $\mathrm{w}$ et avec le y celui de w et $\mathrm{y}$, ce qui est lourd » (Ibn 'Ușfūr 1970 : 535). À nouveau, sur cette notion de lourdeur, voir Bohas \& Lowenstamm (à paraître). On obtient ainsi yaġzuw et yarmiy (= yagzū et yarmī). Pour hašiya/yahšs", la représentation initiale sera yahšayu (yaf"alu) et MUTA1 s'appliquera pour donner yaȟsa" (= yaȟ̌s $\bar{a})$.

Ensuite, comme le dit Abū Ḥayyān dans le Mubdi (qui est un résumé du Mumti) :

Si l'on met le ressemblant en relation prédicative avec le 'alif qui est le pronom du duel ou le $w \bar{a} w$ qui est le pronom du pluriel masculin ou le $n$ qui est le pronom du pluriel féminin, son statut $(h u k m)$ est le même que celui de son passé quand il est mis en relation prédicative avec l'un d'eux. (Abū Ḥayyān 1982 : 202)

Ainsi, yaġzūna est dérivé de yaġzu auquel on ajoute wna ce qui donne

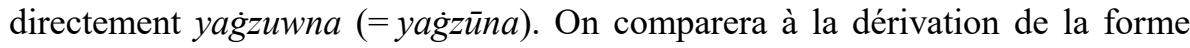
analogue yad 'üna dans la thèse de Brame (1970:106) :

$\begin{array}{ll}\text { /ya }+d^{\prime} u w+u w+n a / & \text { représentation phonologique } \\ \text { ya }+d^{\prime} u \emptyset+u w+n a & \text { Glide Elision } \\ \text { ya }+d^{\prime} u+\varnothing w+n a & \text { Truncation } \\ \text { ya }+d^{\prime} u+u+n a & \text { Syl. Assim } \\ \text { ya }+d^{\prime} \bar{u}+\text { na } & \text { Lengthening } \\ \text { yad'üna } & \end{array}$

La règle Syllabicity assimilation est ainsi formulée (ibid. : 97 et 134) :

$$
\left\{\begin{array}{c}
w \\
y
\end{array}\right\} \rightarrow\left\{\begin{array}{c}
u / u \\
i / i
\end{array}\right\}-\left\{\begin{array}{l}
\# \\
C
\end{array}\right\}
$$

Quant à yahšawna, elle est dérivée de la forme du singulier yahšs̆a à laquelle on ajoute wna : yahš $\bar{a}$ " wna où l'on observe la succession de deux quiescentes qui est résolue par l'élimination de la première (le alif) : yahš̄āØwna. On comparera avec la dérivation de la forme analogue yalqawna chez Brame (ibid. : 101) :

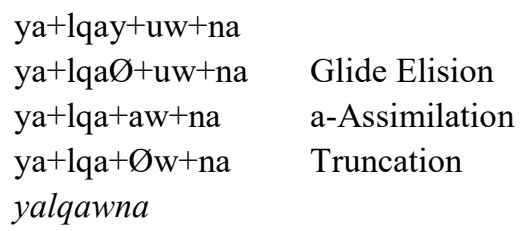


La règle a-Assimilation est formulée ainsi (ibid. : 134) :

$$
\left\{\begin{array}{l}
u \\
i
\end{array}\right\} \rightarrow a / a-
$$

Ces exemples devraient suffire pour montrer la différence d'organisation des deux modèles, que l'on peut confirmer encore par l'étude du participe passif qui, selon les GA, est dérivé du « ressemblant» (voir Bohas 1979 : 226 ; Bohas \& Guillaume 1984 : 154).

Pour un participe passif pluriel de forme VIII : muștafawna, dans la perspective de Brame on posera la représentation sous-jacente : /muștafay+uw+na/. Rien de pareil pour les GA. Muṣtafawna est dérivé du singulier muștafa" (=muștafā), lui-même issu de la représentation analogique: muștafayu par MUTA1.

Représentation analogique : mușțafayu

MUTA1 : mustafa" qui est la forme du singulier

Adjonction du pluriel wna ou yna :

muștafa"wna muștafa"yna

Interdiction des 2 quiescentes $* 2 \mathrm{Q}>$ effacement du $"$ :

muștafawna muștafayna

Le texte d'Ibn al-Sarrāğ, La grammaire résumée (1965 : 98), était déjà tout à fait clair à ce sujet :

Si tu pluralises le défectueux masculin en ajoutant wn ou yn, tu effaces le alif et tu laisses le a qui le précède en l'état, et tu dis pour muștafa" : muștafawna et pour un homme que tu appelles qafa", qafawna.

Fort logiquement, pour le cas indirect la forme est obtenue de la même manière : muștafa" > muștafa"yna > muștafayna.

Ibn al-Sarrağ étant mort en 928, cela montre que cette organisation a été mise en place dès les débuts du modèle des grammairiens arabes.

\section{CONCLUSION}

L'approche générative standard, telle qu'elle est pratiquée dans la thèse de Brame, et l'approche des grammairiens arabes ont en commun les points suivants: l'explication des formes incluant des consonnes « malades ${ }^{16} w$ ou $y$ nécessite de poser une représentation abstraite à laquelle s'appliquent des règles. Ces règles opèrent des transformations : effacement, permutation. Dans les deux cadres ces règles sont générales. Ce point a été discuté par Ibn Ğinnī dans les Haṣā'iș (I : 169) : «Une règle qui n'est pas générale n'est pas correcte. » Dans la grammaire générative ces règles sont markoviennes, pour les GA, au contraire, ces règles peuvent prendre en compte les niveaux antérieurs ou ultérieurs. La plus grande différence tient au fait que pour les générativistes, qui ont le concept de morphème, ces représentations phonologiques comportent simultanément tous les 
morphèmes qui les composent, par exemple, pour katabatā : /katab+at $+\bar{a} /$, pour yad'üna: /ya+d'uw+uw+na/.

Rien de pareil pour les GA. Ne disposant pas du concept de morphème, ils procèdent de mot à mot. Pour yad 'üna, par exemple, ils partent du mot singulier yad'uw $\left(=y^{\prime} d^{\prime} \bar{u}\right)$ dont la représentation analogique est yad uwu comme yaktubu. À cette représentation analogique s'applique la règle d'effacement : « On efface le u car il est ressenti comme lourd sur le y et le w, car avec le w ils ont le statut de deux w et avec le y celui de w et $\mathrm{y}$, ce qui est lourd. » Ce qui donne yad 'uw $\left(=y a d^{\prime} \bar{u}\right)$, à cette forme phonétique, on ajoute le pronom w du pluriel avec le na flexionnel, ce qui donne yad'uwwna où se réalise une séquence de deux quiescentes (les deux w); on en élimine un et il reste yad'uwna (=yad'üna) qui est bien la forme phonétique attestée.

Ce mélange des formes concrètes qui deviennent des représentations abstraites pour d'autres formes concrètes est bien évidemment complètement étranger à la grammaire générative, mais c'est le prix à payer pour l'absence du concept grammatical de morphème. D'autre part, ce qui n'existe pas dans la thèse de Brame, l'application des règles doit être justifiée par de grands principes : ${ }^{2} \mathrm{Q}$ et *4CV par exemple, ou par référence à l'intuition du locuteur qui trouve « lourdes » certaines séquences et applique en conséquence les règles pour les alléger. On est bien loin de l'application mécanique des règles ordonnées du cadre standard. Néanmoins, si l'on prend en compte l'organisation générale en deux niveaux : phonologique et phonétique, reliés par des règles, Brame a eu raison de dire qu'il se situait « in the spirit of the Arab grammarians $»^{17}$.

\section{BIBLIOGRAPHIE}

\section{Textes des grammairiens arabes}

Abū Ḥayyān al-Naḥ̂ī al-Andalūsī. 1982. Al-Mubdi 'fì l-taṣrīf, éd. par 'Abd al-Ḥamīd al-Sayyid Țalb. Koweït : Dār al-'Urūba lil-našr wal-tawzī' .

Ibn Ğinnī, Abū l-Fatḥ 'Uțmān. 1913. Al-Taṣrīf al-Mulūkī, éd. par Muḥammad Sa īe b. Muștafāa an-Na'sān al-Hamwī. Le Caire : Mațba' at Šarikat al-Tamaddun alȘinā iyya.

Ibn Ğinnīe Abū l-Fatḥ 'Uțmān. 1952. Al-Hașā iș, éd. par Muḥammad 'Alī al-Nağğāar. Beyrouth : Dār al-Hudā.

Ibn Ğinnī, Abū l-Faṭ̣ 'Uțmān. 1960. Al-Munșif, Šarh Kitāa al-Taṣrīf li-l-Māzinī, éd. par Ibrāhīm Muștafâ, 'Abdallah Amīn. 3 vol. Le Caire : Muștafā al-Bābī al-Halabī.

Ibn al-Haāğib, in al-Astarābādīi, Rāộ̄ l-ḍīn Muhammad b. al-Ḥasan. 1975 [1939]. Šarh al-Š́ăfiya, éd. par Nūr al-Ḥasan, 'Abd al-Ḥamīd et al-Zafzāf. 4 vol. Beyrouth : Dār al-kutub al-'ilmiyya [publication originale : Le Caire : al-Maktaba al-Tijāriyya alKubrā. 1939].

Ibn al-Sarrāğ, Abū Bakr Muhammad b. Sirrī. 1965. Al-Mūğaz fì n-naḩw, éd. par Moustafa al-Chouémi et Bensalem Damerdji. Beyrouth : A. Badran \& Co.

Ibn 'Ușfūr, Abu l-Ḥasan 'Alī. 1970. Al-Mumti' fĭ l-Tașrīf, éd. par Fah̆r al-Dīn Qabāwa. 2 vol. Alep : Al-Maktaba al-'arabiyya.

17 Brame (1970 : VII). 
Ibn Ya'ī̌s, Muwaffaq al-Dīn Ya išs. 1886. Šarḥ al-Mufașsal, vol. 2, éd. par Gustav Jahn. Leipzig : E. A. Brockhaus.

Ibn Ya'īš, Muwaffaq al-Dīn Ya'iš. S. d. Šarh al-Mufașșal, vol. 10. Le Caire : alȚibā'a al-munīriyya.

Ibn Ya '̌řs, Muwaffaq al-Dīn Ya iš. 1973. Šarh al-Mulūkī fì l-Taṣrīf, éd. par Faḩr alDīn Qabāwa. Alep : Al-Maktaba al-'Arabiyya.

al-Ushmūni, Nūr al-ḍinn Abū 1-Ḥasan. S. d. In Hāšriyat al-Ṣabbān (Muhammad b. 'Alī) 'alā šarḥ al-Ušmūnī 'alā àlfiyyat ībn Mālik, 4 vol. Le Caire : Dār iḥyā' al-kutub al-'arabiyya.

\section{Autres}

Blachère, Régis \& Maurice Gaudefroy-Demombynes. 1975 [1937]. Grammaire de l'arabe classique. Paris : Maisonneuve et Larose.

Bohas, Georges. 1979. Contribution à l'étude de la méthode des grammairiens arabes en morphologie et en phonologie, d'après des grammairiens arabes " tardifs" . Thèse de doctorat d'État. Paris : université Paris III.

Bohas, Georges \& Jean-Patrick Guillaume. 1984. Études des théories des grammairiens arabes. I : Morphologie et phonologie. Damas : Institut français de Damas.

Bohas, Georges \& Jean Lowenstamm. À paraître. "The tașrīf in the Medieval Arabic Grammatical Tradition".

Bohas Georges \& Bruno Paoli. 1997. Aspects formels de la poésie arabe. Toulouse : AMAM.

Brame, Michael K. 1970. Arabic Phonology: Implications for Phonological Theory and Historical Semitic. Thèse de doctorat. Cambridge : MIT.

Cantineau, Jean. 1950a. Racines et schèmes. Mélanges William Marçais, Institut d'études islamiques de l'Université de Paris. Paris : Maisonneuve. 119-124.

Cantineau, Jean. 1950b. La notion de schème et son altération dans diverses langues sémitiques. Semitica 3 : 73-83.

Chomsky, Noam \& Morris Halle. 1968. The Sound Pattern of English. New York, Evanston \& London : Harper and Row.

Duval, Rubens. 1881. Traité de grammaire syriaque. Paris : Vieweg.

Kiparsky, Paul. 1973. How Abstract is Phonology? Three Dimensions in Linguistic Theory, dir. par O. Fujimura, D. L. Smith, S. I. Harada, \& J. B. Lovins. Tokyo : Tokyo Institute for Advanced Studies of Language. 5-56.

Lightner, Theodore M. 1979. On the English Etymology of STAR. Linguisticae Investigationes 3(2) : 285-293.

McCarthy, John J. 1979. Formal Problems in Semitic Phonology and Morphology. Thèse de doctorat. Cambridge : MIT.

Sartori, Manuel. 2017. Manuel de conjugaison du verbe en arabe. Marseille: Diacritiques éditions.

Touzard, Jean. 1949 [1905]. Grammaire hébräque abrégée. Paris : Jean Gabalda.

Wright, William. 1971 [1859-1862]. A Grammar of the Arabic Language. Cambridge : Cambridge University Press. 